\title{
Rational functions with given ramification in characteristic $p$
}

\author{
Brian Osserman
}

\begin{abstract}
Using limit linear series and a result controlling degeneration from separable maps to inseparable maps, we give a formula for the number of rational functions (up to automorphism of the target) on the projective line with ramification to order $e_{i}$ at general points $P_{i}$, in the case that all $e_{i}$ are less than the characteristic. Unlike the case of characteristic 0 , the answer is not given by Schubert calculus, nor is the number of maps always finite for distinct $P_{i}$, even in the tamely ramified case. However, finiteness for general $P_{i}$, obtained by exploiting the relationship to branched covers, is a key part of the argument.
\end{abstract}

\section{Introduction}

We work throughout over an algebraically closed field $k$ of characteristic $p$. For convenience, and because our results will be trivial in the case $p=2$, we assume $p>2$ throughout. The question we wish to address in this paper is simply the following.

Question 1.1. Fix $n$ points $P_{i}$ on $\mathbb{P}^{1}$ and integers $e_{i} \geqslant 2$, with $\sum_{i}\left(e_{i}-1\right)=2 d-2$, and $e_{i} \leqslant d$ for all $i$. How many separable self-maps of $\mathbb{P}^{1}$ of degree $d$ are there which ramify to order $e_{i}$ at the $P_{i}$, counted modulo automorphism of the image $\mathbb{P}^{1}$ ?

Here, note that the ramification points are fixed on the source, and not on the target. The condition that $\sum_{i}\left(e_{i}-1\right)=2 d-2$ implies, by the standard characteristic- $p$ Riemann-Hurwitz formula, that there are no solutions if any of the $e_{i}$ are not prime to $p$, so we will assume throughout that all $e_{i}$ are prime to $p$ unless we specify otherwise.

In characteristic 0, the number of maps is always finite, and when the $P_{i}$ are general, this number is given combinatorially in terms of Schubert calculus (see, e.g., [Oss03]). In characteristic $p$, neither of these statement holds in general, and we explore the situation in a complete range of characteristics, showing that the situation can be particularly pathological in low characteristics regardless of whether the ramification is tame or wild, and ultimately solving the problem in midrange and higher characteristics (see Definition 1.3 below). Immediate motivation for this paper was given by surprising applications to logarithmic connections with vanishing $p$-curvature on $\mathbb{P}^{1}$, and consequently Frobenius-unstable vector bundles on curves of genus 2, as well as the geometry of the generalized Verschiebung. Specifically, in [Oss04d], logarithmic connections on rank 2 vector bundles on $\mathbb{P}^{1}$ with vanishing $p$-curvature are related via study of their subsheaf of horizontal sections to the rational functions studied here. In [Oss04a], degenerations are used to phrase certain questions about rank 2 Frobenius-destabilized vector bundles on genus 2 curves in terms of the connections on $\mathbb{P}^{1}$ analyzed in [Oss04d], and this in turn is used to study the action of pullback under

Received 27 July 2004, accepted in final form 25 September 2005.

2000 Mathematics Subject Classification 14N10.

Keywords: rational function, prescribed ramification, positive characteristic.

This paper was partially supported by fellowships from the National Science Foundation and Japan Society for the Promotion of Science.

This journal is (C) Foundation Compositio Mathematica 2006. 


\section{B. OSSERman}

Frobenius on the moduli space of rank 2 bundles and trivial determinant in [Oss04b]. However, the main question addressed is sufficiently fundamental that a range of applications may be expected; in characteristic 0, a still-open generalization of this question to a higher-dimensional target is applicable to the solution of the $A_{N}$ Bethe equation of XXX type (see [MV02]). In addition, the results and techniques of this paper lead to a number of new results on the existence and nonexistence of branched covers of the projective line; see [Oss05b].

There is considerable literature on our main question and its natural generalizations in characteristic 0, from Eisenbud and Harris' original solution in the case of $\mathbb{P}^{1}$ in [EH83, Theorem 9.1], to combinatorial formulas in the same cases by Goldberg [Gol91] and Scherbak [Sch02], to formulas in the higher genus case of Logan [Log03, Theorem 3.1] and the author [Oss03]. However, the present work appears to be the first attempt to approach the problem for positive characteristics.

Our basic technique is an adaptation of the limit linear series degeneration argument of [Oss03], solving the problem first in the case of three points, and then repeatedly letting ramification points come together to reduce inductively to this case. The main obstruction to carrying this argument through is controlling potential degeneration of separable maps to inseparable maps.

We now give some notation and terminology leading up to the statement of our main theorem.

Notation 1.2. When the answer to Question 1.1 is finite, we denote it by $N\left(\left\{\left(P_{i}, e_{i}\right)\right\}_{i}\right)$. We denote by $N_{\text {gen }}\left(\left\{e_{i}\right\}_{i}\right)$ the value of $N\left(\left\{\left(P_{i}, e_{i}\right)\right\}_{i}\right)$ for general $P_{i}$.

Definition 1.3. We distinguish three ranges of characteristic. We will refer to the high characteristic range to mean those characteristics for which $p>d$, as well as characteristic 0 . The mid characteristic range will be characteristics for which $p \leqslant d$, but $e_{i}<p$ for all $i$. Finally, the low characteristic range will be characteristics for which $p \leqslant e_{i}$ for some $i$.

We will see that high characteristics are uniformly well-behaved with respect to our question, while low characteristics can be extremely pathological, and the mid characteristics seem to be reasonably well-behaved, but are considerably subtler than the high characteristics.

THEOREM 1.4. In the mid and high characteristics, we have the following complete solution to our main question:

$$
\begin{gathered}
N_{\text {gen }}\left(e_{1}, e_{2}, e_{3}\right)= \begin{cases}1 & p>d \\
0 & \text { otherwise }\end{cases} \\
N_{\text {gen }}\left(\left\{e_{i}\right\}_{i}\right)=\underset{\substack{d-e_{n-1}+1 \\
d-e_{n}+1} \leqslant d^{\prime} \leqslant \underset{p+d-e_{n-1}-e_{n}}{d}}{N_{\text {gen }}\left(\left\{e_{i}\right\}_{i \leqslant n-2}, e\right),} \text { with } e=2 d^{\prime}-2 d+e_{n-1}+e_{n}-1 .
\end{gathered}
$$

Equivalently, for $n>3, N_{\text {gen }}\left(\left\{e_{i}\right\}_{i}\right)$ is given as the number of $(n-3)$-tuples of positive integers $e_{2}^{\prime}, \ldots, e_{n-2}^{\prime}$ such that any consecutive triple $e, e^{\prime}, e^{\prime \prime}$ of the sequence

$$
e_{1}, e_{2}, e_{2}^{\prime}, e_{3}, e_{3}^{\prime}, \ldots, e_{n-2}, e_{n-2}^{\prime}, e_{n-1}, e_{n},
$$

with $e=e_{1}$ or some $e_{i}^{\prime}$, satisfies the following properties:

(i) the sum $e+e^{\prime}+e^{\prime \prime}$ is odd, and less than $2 p$;

(ii) the triple $e, e^{\prime}, e^{\prime \prime}$ satisfies the triangle inequality: i.e., $e \leqslant e^{\prime}+e^{\prime \prime}, e^{\prime} \leqslant e+e^{\prime \prime}$, and $e^{\prime \prime} \leqslant e+e^{\prime}$.

Further, for general points $P_{i}$ all of the relevant maps have no non-trivial first-order deformations.

Remark 1.5. We make a few observations about the recursive formula: first, as the degree $d^{\prime}$ is always no greater than $d$, high characteristic will remain high under recursion. Similarly, adding the two inequalities on the right, we find $e=2 d^{\prime}-2 d+e_{n}+e_{n-1}-1 \leqslant d+\left(p+d-e_{n-1}-e_{n}\right)-2 d+e_{n}+e_{n-1}-1=$ $p-1$, so mid characteristic is also preserved (or becomes high) under iteration. 


\section{RATIONAL FUNCTIONS WITH GIVEN RAMIFICATION IN CHARACTERISTIC $p$}

Note that in the high characteristic range, we always have $p>d$, so the answer becomes independent of characteristic: this is visibly true for the $n=3$ formula, and is true for the recursive formula because the inequality $d^{\prime} \leqslant p+d-e_{n-1}-e_{n}$ is subsumed by the inequality $d^{\prime} \geqslant e=2 d^{\prime}-2 d+e_{n}+e_{n-1}-1$, or equivalently $d^{\prime} \leqslant 2 d-e_{n-1}-e_{n}+1$, which is necessary for the number of maps $N_{\text {gen }}\left(\left\{e_{i}\right\}_{i \leqslant n-2}, e\right)$ to be non-zero. Unsurprisingly, this characteristic-independent formula is also the answer in characteristic 0 .

Finally, we remark that although the recursive formula reflects the degeneration used in the proof of the theorem, the second formula is easier to analyze combinatorially, and in particular it is easier to use this form to prove positivity for a given choice of $e_{i}$. This may also be pictured in terms of a degeneration, to a chain of smooth rational curves with a total of three ramification points or nodes on each component, but it seems difficult to prove the main theorem directly in terms of this degeneration.

We also remark that chronologically, the direct approach here was not the first proof discovered of our formulas. That was obtained via correspondence with certain logarithmic connections on $\mathbb{P}^{1}$ together with a theorem of Mochizuki, as outlined in [Oss04e]. The key step of the direct argument presented here, the analysis of separable maps degenerating to inseparable maps, was derived via a careful study of the corresponding situation with connections.

We begin in $\S 2$ by translating the problem into a question on intersection of Schubert cycles in a Grassmannian. We exploit the relationship between ramified maps and branched covers in $\S 3$ to obtain some basic finiteness results including a ramified Brill-Noether-type theorem for $g_{d}^{1}$ on $\mathbb{P}^{1}$ with specified ramification. We then apply this in $\S 4$ to solve the base case of three ramification points. Section 5 appears at first blush to be merely a couple of eccentric observations, including the pathology that when exactly one $e_{i}$ is greater than $p$, the number of maps can never be finite, but these observations play key roles in $\S 6$, where we give a precise analysis of when a family of separable maps can degenerate to an inseparable map, and in $\S 7$, where we finally prove our main theorem via the degeneration argument using limit linear series. Finally, in $\S 8$ we explore some examples and further questions, and in the Appendix we construct a scheme representing maps between a pair of fixed curves, with at least a certain specified ramification, but at points which are allowed to move; this scheme is the key idea in the proof of the Brill-Noether-type result of $\S 3$, and is also used to generalize this result in [Oss05a].

The contents of this paper form a portion of the author's 2004 PhD thesis at MIT, under the direction of Johan de Jong.

\section{Translation to Schubert cycles}

In this section, we translate Question 1.1 into a question on intersection of Schubert cycles on the projective Grassmannian $\mathbb{G}(1, d)$, and pin down some related notation. The translation is easy enough: a map (up to automorphism of the image) may be represented explicitly by a two-dimensional space of polynomials of degree $d$. Following standard linear series notation, we will refer to a point of our Grassmannian as a ' $g_{d}^{1}$ '. It is then easy to see that a ramification condition of order $e$ at a point $P$ corresponds to a Schubert cycle of codimension $e-1$, which we denote by $\Sigma_{e-1}(P)$. As we assumed $\sum_{i}\left(e_{i}-1\right)=2 d-2$, and our Grassmannian has dimension $2 d-2$, the expected dimension of the intersection is therefore 0. Pieri's formula will now give us the intersection product of our cycles, yielding a hypothetical formula for the answer to our question. However, there are several substantive issues to address.

The first major issue is whether or not the Schubert cycles will actually intersect transversely, even for general choice of the $P_{i}$. Vakil [Vak03, Corollary 2.7(a)] and Belkale [Bel06, Theorem 0.9] 


\section{B. OSSERMAN}

have recently independently shown that if the Schubert cycles are general, they will intersect transversely, but it is not the case that general points on $\mathbb{P}^{1}$ will correspond to general Schubert cycles in $\mathbb{G}(1, d)$, so we cannot hope to apply such general results. In fact, they correspond to osculating flags of the rational normal curve in $\mathbb{P}^{d}$. In characteristic 0 , properness of the intersection (that is, having the expected dimension) for any choice of distinct $P_{i}$ is straightforward, and we will reproduce the argument below in order to analyze its implications in characteristic $p$. Transversality for a general choice of $P_{i}$ in characteristic 0 is known, but more involved (see [EH83, Theorem 9.1]), and means that Pieri's formula actually yields the correct number for general choice of $P_{i}$. However, all of these statements fall apart in characteristic $p$, as we will see shortly.

The second issue to face is that of base points: points of $\mathbb{G}(1, d)$ with base points correspond to lower degree maps padded out by extra common factors in the defining polynomials. It is easy to see by Riemann-Hurwitz that base points cannot occur for separable maps. In particular, in characteristic 0, or when $p>d$, the intersection of our Schubert cycles always actually corresponds to the desired $g_{d}^{1}$ 's. On the other hand, in general inseparable maps can and will occur, frequently contributing an excess intersection. For instance, in the case $d>p, e_{i}<p$, the Frobenius map will always contribute a $\mathbb{P}^{d-p}$ to the intersection, with one point in $\mathbb{G}(1, d)$ for every choice of a degree $d-p$ base point divisor.

These are the two issues which must be addressed in order to give an answer to the question. However, except in the base case of three points, we will not address them directly, as would be required by an intersection-theoretic approach. We will rather take a different tack, looking at moduli of $g_{d}^{1}$ 's with specified ramification for certain degenerating families. Before continuing, we summarize as follows.

Proposition 2.1. The answer to Question 1.1 is given by the number of points corresponding to separable maps inside the intersection $\bigcap_{i} \Sigma_{e_{i}-1}\left(P_{i}\right) \subset \mathbb{G}(1, d)$ of the Schubert cycles $\Sigma_{e_{i}-1}\left(P_{i}\right)$.

Warning 2.2. The equivalences between maps and linear series tend to become misleading in families; in particular, if we have a linear series which develops base points in a special fiber, there is no way to remove them globally to actually produce a morphism. For our arguments, whenever we are working over a base other than a field, we will always be dealing with linear series, even if we describe it as a 'family of maps'. For the appropriate definitions (albeit in an overly generalized context), see [Oss04c].

\section{Finiteness results}

We begin with a proposition whose argument is well-known in characteristic 0 :

Proposition 3.1. In any characteristic, if $\sum_{i}\left(e_{i}-1\right)=2 d-2-c$ for some $c \geqslant 0$, then any component of $\bigcap_{i} \Sigma_{e_{i}-1}\left(P_{i}\right)$ having dimension greater than $c$ must meet the inseparable locus. In particular, in high characteristics, $\bigcap_{i} \Sigma_{e_{i}-1}\left(P_{i}\right)$ always has the expected dimension $c$.

Proof. By Riemann-Hurwitz, if we had $\sum_{i}\left(e_{i}-1\right)>2 d-2$, then $\bigcap_{i} \Sigma_{e_{i}-1}\left(P_{i}\right)$ must consist entirely of inseparable maps. One can then induct on $c$, with a base case of $c=-1$, and the induction step consisting of imposing a simple ramification condition at a new point $P$. This is a codimension 1 condition, and we claim it meets every positive-dimensional component of $\bigcap_{i} \Sigma_{e_{i}-1}\left(P_{i}\right)$ : indeed, the condition it imposes inside $\mathbb{G}(1, d)$ is that the lines meet a given $(d-2)$-plane in $\mathbb{P}^{d}$, and any positive-dimensional subscheme of $\mathbb{G}(1, d)$ corresponds to a variety of dimension at least 2 in $\mathbb{P}^{d}$, so at least one line in the variety meets the $(d-2)$-plane. Thus, each component of dimension at least $c+1$ produces a component of dimension at least $c$ in the case of expected dimension $c-1$, so by induction, both components contain inseparable maps. 


\section{RATiOnAL FUnCTIONS WITH GIVEN RAMIFICATION IN CHARACTERISTIC $p$}

The case $c=0$ is simply the full specification of a tame ramification divisor, so we restate as follows.

COROLlary 3.2. In high characteristics, there are only finitely many self-maps of $\mathbb{P}^{1}$ with specified tame ramification divisor.

The finite generation of fundamental groups of curves, together with some generalities on existence of moduli spaces of maps with certain ramification behavior, gives us a more substantive finiteness result than the previous proposition.

Theorem 3.3. Let $e_{i}$ be prime to $p$, and suppose that $\sum_{i}\left(e_{i}-1\right)=2 d-2$. Then for a general choice of points $P_{i}$, we have that the set of maps from $\mathbb{P}^{1}$ to $\mathbb{P}^{1}$ ramified to order $e_{i}$ at $P_{i}$, modulo automorphism of the image:

(i) is finite;

(ii) has no elements mapping any two of the $P_{i}$ to the same point.

Proof. By Theorem A.6, we have a moduli scheme $M R=M R^{d}\left(\mathbb{P}^{1}, \mathbb{P}^{1},\left\{e_{i}\right\}_{i}\right)$, with ramification and branching maps down to $\left(\mathbb{P}^{1}\right)^{n}$, and actions of Aut $\left(\mathbb{P}^{1}\right)$ on both sides, with the action on the domain being free. It is well-known that given any specified tame branch locus, up to automorphism of the cover there are only finitely many covers with the given degree and branching: this follows, for instance, from the finite generation of the tame fundamental group of $\mathbb{P}^{1}$ minus the branch points.

Thus, each fiber of the branch morphism branch $: M R \rightarrow\left(\mathbb{P}^{1}\right)^{n}$ has only finitely many Aut $\left(\mathbb{P}^{1}\right)$ orbits, and is therefore of dimension at most $\operatorname{dim} \operatorname{Aut}\left(\mathbb{P}^{1}\right)=3$. We conclude that the dimension of $M R$ is at most $n+3$. This immediately implies that a general fiber (in the sense of a fiber above a general point of $\left(\mathbb{P}^{1}\right)^{n}$, making no hypotheses on dominance) of the ramification morphism ram $: M R \rightarrow\left(\mathbb{P}^{1}\right)^{n}$ can have dimension at most 3 . By the freeness of the Aut $\left(\mathbb{P}^{1}\right)$-action on this side, this completes the proof of part (i).

The proof of part (ii) proceeds similarly: one sees that the locus $M R^{\prime}$ of maps in $M R$ sending any two ramification points to the same branch point has dimension at most $n-1+3=n+2$, and the fibers of $M R^{\prime}$ under the ramification morphism are still Aut $\left(\mathbb{P}^{1}\right)$-orbits, so we conclude that a general fiber of the ramification morphism cannot contain any points of $M R^{\prime}$, completing the proof.

Remark 3.4. This finiteness theorem may be considered a first case in positive characteristic of a Brill-Noether theorem with prescribed ramification, as in [EH86, Theorem 4.5]. We show via deformation theory of covers in [Oss05a] that one can generalize further in the $r=1$ case, which gives in particular an intrinsically algebraic and characteristic- $p$ proof of the previous theorem.

\section{The case of three points}

While the general problem we wish to study becomes rather subtle in characteristic $p$, the special case where we only have three ramification points is more tractable. This is fortuitous, as this case will form the base case of our general induction argument. We begin by observing that in this case, as each ramification index must be at most $d$, all three ramification points must map to distinct points. We can also show the following via elementary observations.

LEMma 4.1. The intersection $\bigcap_{i} \Sigma_{e_{i}-1}\left(P_{i}\right)$ for three points is scheme-theoretically isomorphic to $\mathbb{P}^{m}$ for some $m \geqslant 0$.

Proof. Set $P_{1}=0, P_{2}=\infty$, and $P_{3}=1$, and simultaneously fix bases $(F, G)$ (up to simultaneous scaling) for our $g_{d}^{1}$ 's by requiring that $F$ vanish at $0, G$ vanish at $\infty$, and $F(1)=G(1)$. We may then verify the assertion directly by looking at the conditions imposed on the coefficients of $F$ and $G$ by the ramification conditions. 


\section{B. OSSERMAN}

We now show the following.

Theorem 4.2. Let $P_{1}, P_{2}, P_{3}$ be three distinct points of $\mathbb{P}^{1}$, and $e_{1}, e_{2}, e_{3}$ positive integers. Then we have the following.

(i) In any characteristic, $N\left(\left\{\left(P_{i}, e_{i}\right)\right\}_{i}\right)$ is finite, and is in fact always 0 or 1 , being 0 if and only if there is some inseparable $g_{d}^{1}$ of degree $d$ with the required ramification. Moreover, when $N\left(\left\{\left(P_{i}, e_{i}\right)\right\}\right)=1$, the intersection is actually given scheme-theoretically by a single reduced point.

(ii) Whenever $e_{1}$ and $e_{2}$ are less than $p$ and $d \geqslant p$, we have $N\left(\left\{\left(P_{i}, e_{i}\right)\right\}_{i}\right)=0$. Whenever $d<p$, we have $N\left(\left\{\left(P_{i}, e_{i}\right)\right\}_{i}\right)=1$.

Proof. We first deduce (ii) from (i): the second claim of (ii) is trivial, as if $d<p$, there can be no inseparable map of degree $d$. For the first claim, because $e_{1}$ and $e_{2}$ are both less than $p$, any inseparable map will satisfy the required ramification conditions at $P_{1}$ and $P_{2}$, and if we choose our map to be Frobenius, we can check directly that by adding base points at $P_{3}$ we can satisfy the last ramification condition as well.

For the proof of (i), we begin by noting that the intersection product in question is always 1 : indeed, as all of the Schubert cycles in question are special, this follows immediately by applying Pieri's formula and then the complementary-dimensional cycle intersection formula (see [Ful98, Duality theorem, p. 271]). Next, the separable locus must be finite, as for three points on $\mathbb{P}^{1}$ there are no moduli, so we can apply Theorem 3.3. Finally, by Lemma 4.1, our intersection is a $\mathbb{P}^{m}$, and is in particular connected. If it is zero-dimensional, we are done, as we get a single reduced point which must clearly correspond to either a separable or inseparable map. On the other hand, if it is positive dimensional, by the closedness of the inseparable locus and the finiteness of the separable locus, we find that all the maps are inseparable.

To rephrase a slightly special case of the second part of the theorem, we have the following.

Corollary 4.3. Suppose we are in the situation of the preceding theorem, and $e_{1}, e_{2}<p$. Then a separable map of the specified ramification exists if and only if $d<p$.

Remark 4.4. This corollary certainly does not hold if we drop the hypothesis that at least two ramification indices be less than $p$, as may be seen by considering the example of $x^{n}$.

\section{Some theorems and pathologies}

In this section, we make observations on what happens when some $e_{i}$ are replaced by $p-e_{i}$, and we also find that when exactly one $e_{i}$ is greater than $p$, there can never be a finite number of maps with the specified ramification.

Notation 5.1. Let $f$ be a separable map $f$ between smooth proper curves $C$ and $D$. Then the different $\delta$ of $f$ is defined to be the divisor on $C$ associated to the skyscraper sheaf obtained as the cokernel of the natural map $f^{*} \Omega_{D}^{1} \hookrightarrow \Omega_{C}^{1}$.

We have the following amusing and occasionally useful lemma.

Lemma 5.2. Fix $e_{i}$ all less than $p$, and points $P_{i}$ on $\mathbb{P}^{1}$. Given an $f$ ramified to order $e_{i}$ at $P_{i}$, and with $f\left(P_{1}\right) \neq f\left(P_{2}\right)$, we can associate an $\hat{f}$ ramified to order $p-e_{i}$ at $P_{i}$ for $i=1,2$ and $e_{i}$ at $P_{i}$ for $i>2$, and with $\hat{f}\left(P_{1}\right) \neq \hat{f}\left(P_{2}\right)$. This association is defined uniquely on equivalence classes modulo automorphism of the image $\mathbb{P}^{1}$, and induces a bijection on such equivalence classes.

In particular, if $e_{i}^{\prime}$ are any integers obtained from the $e_{i}$ by repeatedly replacing pairs of indices $e_{i}, e_{j}$ with $p-e_{i}, p-e_{j}$ while holding the others fixed, we have $N_{\text {gen }}\left(\left\{e_{i}\right\}_{i}\right)=N_{\text {gen }}\left(\left\{e_{i}^{\prime}\right\}_{i}\right)$. 


\section{RATIONAL FUNCTIONS WITH GIVEN RAMIFICATION IN CHARACTERISTIC $p$}

Proof. For convenience, we assume that $f$ is unramified at infinity. By composing $f$ with an automorphism of the image $\mathbb{P}^{1}$, we may write it (uniquely up to scalar) as $F / G=\left(x-P_{1}\right)^{e_{1}} F^{\prime} /\left(x-P_{2}\right)^{e_{2}} G^{\prime}$. If we multiply through by $\left(x-P_{2}\right)^{p} /\left(x-P_{1}\right)^{p}$, we get the new function $\hat{f}=\left(x-P_{2}\right)^{p-e_{2}} F^{\prime} /$ $\left(x-P_{1}\right)^{p-e_{1}} G^{\prime}$. As we obtained it from the old function by multiplying by an inseparable function, one checks that the different is unaffected away from $P_{1}$ and $P_{2}$. As we assumed all $e_{i}<p$, it follows that the new function and old function have the same ramification away from $P_{1}$ and $P_{2}$, and a priori infinity. On the other hand, it is clear that the ramification at $P_{1}$ and $P_{2}$ is now $p-e_{1}$ and $p-e_{2}$, and it is easy to check that the new degree of the function allows for no new ramification at infinity. This operation is visibly invertible and well-defined up to automorphism equivalence, and in particular gives the desired bijection.

For the second statement, we just induct on pairs of $e_{i}, e_{j}$, making use of the fact that by Theorem 3.3, for $P_{i}$ general, none of our maps for either of the two relevant choices of ramification indices send any two of the $P_{i}$ to the same point.

The main usefulness of this rather eccentric fact is summarized in the following, to be applied later on.

Corollary 5.3. To calculate $N_{\text {gen }}\left(\left\{e_{i}\right\}_{i}\right)$ completely in the mid and high characteristic range, it suffices to do so either when all but at most one of the $e_{i}$ are less than $p / 2$, or when all the $e_{i}$ are odd. Moreover, it suffices to prove Theorem 1.4 in only either of these two cases.

Proof. The first statement follows trivially from the previous corollary. The second is simply a matter of noting that for any given number of points, the parity of the sum of the $e_{i}$ is determined by the integrality of $d$. All the $e_{i}$ being odd always gives the correct parity, and if any $e_{i}$ are even, an even number of them must be.

To get the final assertion, we have to show that the formulas proposed in Theorem 1.4 are unaffected by replacing a pair $e_{i}$ and $e_{j}$ with $p-e_{i}$ and $p-e_{j}$. We will show this by induction, with $n=3$ as the base case. For convenience, we repeat the formulas in question:

$$
\begin{gathered}
N_{\text {gen }}\left(\left\{e_{i}\right\}_{i}\right)=\sum_{\substack{d-e_{n-1}+1 \\
d-e_{n}+1} \leqslant d^{\prime} \leqslant{ }_{p+d-e_{n-1}-e_{n}}}^{N_{\text {gen }}\left(\left\{e_{i}\right\}_{i \leqslant n-2}, e\right),} \quad \text { with } e=2 d^{\prime}-2 d+e_{n-1}+e_{n}-1 \\
N_{\text {gen }}\left(e_{1}, e_{2}, e_{3}\right)= \begin{cases}1 & p>d \\
0 & \text { otherwise. }\end{cases}
\end{gathered}
$$

We begin with the three point case. Taking into account the additional inequalities $e_{i} \leqslant d$ for all $i$, if we substitute $d=\left(e_{1}+e_{2}+e_{3}-1\right) / 2$, we find our inequalities may be rewritten as:

$$
\begin{aligned}
& e_{1}-e_{2}+1 \\
& e_{2}-e_{1}+1
\end{aligned} \leqslant e_{3} \leqslant \begin{gathered}
e_{1}+e_{2}-1 \\
2 p-1-e_{1}-e_{2} .
\end{gathered}
$$

Replacing any two $e_{i}$ by $p-e_{i}$ will simply permute these inequalities.

Next, as the proposed recursive equation is not visibly symmetric in the $e_{i}$, there are three cases to consider: first, $i=n-1, j=n$; second, $i, j<n-1$; and, finally, $i<n-1, j \geqslant n-1$. We first note that when we replace $e_{i}, e_{j}$ by $p-e_{i}, p-e_{j}$, the degree $d$ changes to $d+p-e_{i}-e_{j}$.

In the first case, one checks that under the substitutions, the inequalities for $d^{\prime}$ are simply permuted, and $e$ remains unchanged. For the second and third cases, we will want to write the inequalities for $d^{\prime}$ as equivalent inequalities for $e$. We find

$$
\begin{aligned}
& e_{n}-e_{n-1}+1 \\
& e_{n-1}-e_{n}+1
\end{aligned} \leqslant e \leqslant \begin{gathered}
e_{n}+e_{n-1}-1 \\
2 p-1-e_{n-1}-e_{n} .
\end{gathered}
$$




\section{B. OSSERman}

In particular, these inequalities depend only on $e_{n}$ and $e_{n-1}$, and not on $d$. Hence, in the second case $e$ ranges through the same values after substituting for $e_{i}$ and $e_{j}$, so we only need to use the induction hypothesis to conclude the desired result.

Finally, in the third case we assume for convenience that $i<n-1$ and $j=n$. In this case, when we substitute into our inequalities for $e$, we get

$$
\begin{aligned}
& p-e_{n}-e_{n-1}+1 \\
& e_{n-1}+e_{n}+1-p
\end{aligned} \leqslant e \leqslant \begin{aligned}
& p-e_{n}+e_{n-1}-1 \\
& p-1-e_{n-1}+e_{n}
\end{aligned}
$$

which then gives us that $p-e$ satisfies precisely the same inequalities that $e$ did originally. Thus, each term in the new recursive formula corresponds to a unique term in the old formula by replacing $e$ with $p-e$ and $e_{i}$ by $p-e_{i}$, so once again the induction hypothesis gives us the desired result.

It remains only to note that the reducedness assertion of Theorem 1.4 is preserved as well when $e_{i}, e_{j}$ are replaced by $p-e_{i}, p-e_{j}$. This follows from the observation that the construction in the preceding lemma works equally well over $k[\epsilon]$, so lack of non-trivial first-order deformations is preserved.

We end with a rather surprising observation illustrating that even tame ramification can have very pathological behavior in low characteristics.

Proposition 5.4. Suppose that $e_{1}>p$ but still prime to $p$, and $e_{i}<p$ for all $i>1$. Then if one map exists with ramification $e_{i}$ at $P_{i}$, infinitely many do. In particular, if the $P_{i}$ are general, no maps exist with ramification $e_{i}$ at $P_{i}$.

Proof. Without loss of generality, we may assume that $P_{1}$ is the point at infinity, and that our function maps infinity to infinity, so that it is given by $F / G$, with $\operatorname{deg} F-\operatorname{deg} G=e_{1}$. Now consider the family of functions $F / G-t x^{p}$, where $t \in k$. As $e_{1}>p$, the ramification at infinity is unaffected. On the other hand, since $x^{p}$ is regular away from infinity and inseparable, the different is unchanged on the affine part, and as we assumed that all $e_{i}<p$ for $i>1$, we find that the ramification is unaffected everywhere, giving us an infinite family of maps, clearly not related by automorphism, all with the same ramification. For $P_{i}$ general we know that there can be at most finitely many maps with specified tame ramification by Theorem 3.3, so we conclude that there cannot be any such maps at all.

The following corollary will not be used later, but seems worth mentioning.

Corollary 5.5. Suppose that the $P_{i}$ are general, and we have a map $f$ with $e_{i}\langle p$ for all $i>1$, but $e_{1}=m p$ wild with $m>1$. Then the order of the different of $f$ at $P_{1}$ is greater than $2(m-1) p$.

Proof. If we again put $P_{1}$ at infinity and write $f=F / G$, subtracting off some multiple of $x^{m p}$ will force the degree to drop, and leave all $e_{i}$ for $i>1$ unchanged. The index $e_{1}$ may not drop (this can only happen if the degree of $F$ drops at least $m p$ below the degree of $G$ ), but if it remains wild we can iterate, and as the degree drops each time, $e_{1}$ must eventually become tame. By our proposition, this new tame index, which we denote by $e_{1}^{\prime}$, would have to be less than $p$. If we denote the new degree by $d^{\prime}$, one sees that $d-d^{\prime}>(m-1) p$, considering separately the cases that the degree of $F$ remained greater than $G$ or dropped below that of $G$. The corollary then follows from Riemann-Hurwitz.

Example 5.6. To demonstrate that the statement of Proposition 5.4 is not vacuous, we note that it is not difficult to write down a concrete example. Indeed, the family of functions $x^{p+2}+t x^{p}+x$ is easily seen to satisfy our requirements. 


\section{RATiOnAL FUnCTIONS WITH GIVEN RAMIFICATION IN CHARACTERISTIC $p$}

Remark 5.7. In standard examples of linear series existing only for special configurations in characteristic 0, the space of linear series with the prescribed ramification is supported over a maximal-dimensional subspace of $\mathcal{M}_{g, n}$ or, equivalently, for a general configuration where such a linear series exists, there are only finitely many of them. In particular, in none of the standard examples is the expected dimension non-negative. It is not clear whether or not this must always be the case in characteristic 0 , but here we have an example where this fails to hold in characteristic $p$.

\section{Specialization to inseparable maps}

The ultimate goal will be to solve the map-counting problem in mid and high characteristics by repeatedly letting points come together. The main obstacle to this is understanding when a family of separable maps can have an inseparable map as its limit. We provide an answer to this question which may seem unmotivated, and indeed arose from careful examination of the situation in the very different and at first glance totally unrelated setting of certain connections with vanishing $p$-curvature, as discussed in [Oss04e].

Our main result is the following.

Theorem 6.1. Let $A$ be a discrete valuation ring containing its residue field $k$ and with uniformizer $t$, and $f_{t}$ be a family of maps of degree $d$ from $\mathbb{P}^{1}$ to $\mathbb{P}^{1}$ over Spec $A$ (more precisely, a linear series on $\mathbb{P}_{A}^{1}$ ) whose generic fiber is tamely ramified along sections $P_{i}$ with all $e_{i}<p$, and whose special fiber is inseparable. We further assume that the $P_{i}$ stay away from infinity. Then if the limit of the $P_{i}$ in the special fiber is denoted by $\bar{P}_{i}$, we have the following.

(i) If the $\bar{P}_{i}$ are distinct, they are in a special configuration allowing the existence of separable maps of degree $d+m-1$ ramified to order $e_{i}$ at $\bar{P}_{i}$, and $2 m-1$ at infinity.

(ii) If $\bar{P}_{j}=\bar{P}_{j^{\prime}}$ with $e_{j}+e_{j^{\prime}}<p$, and the other $\bar{P}_{i}$ distinct, then the $\bar{P}_{i}$ are in a special configuration allowing separable maps of degree $d+m-1-b$, ramified to order $e_{i}$ at the $\bar{P}_{i}$ for $i \neq j, j^{\prime}$, to $e_{j}+e_{j^{\prime}}-2 b-1$ at $\bar{P}_{j}=\bar{P}_{j^{\prime}}$, and to $2 m-1$ at infinity.

In either case, $m$ is some integer with $p \leqslant m \leqslant d$, and in the second case $b$ is a non-negative integer less than $\left(e_{j}+e_{j^{\prime}}-1\right) / 2$.

Proof. The main idea of the proof is not dissimilar to the basic operation of applying fractional linear transformations to be able to factor out a power of the uniformizer if one is given a family of maps degenerating to a constant map. However, in this case we will apply a fractional linear transformation with inseparable coefficients; this will behave similarly, but will not preserve the degree of the map, and also does not appear to work readily in nearly the generality of the constant case.

We work for the most part explicitly with pairs of polynomials and their differents, only dealing with common factors at the end to translate to rational functions and ramification indices. We can write $f_{t}$ as $F / G$, with $F, G \in A[x]$, and have no common factors. We denote by $F_{0}$ and $G_{0}$ the polynomials obtained from $F$ and $G$ by setting $t=0$, and by $\bar{F}_{0}$ and $\bar{G}_{0}$ the inseparable polynomials obtained by canceling the common factors of $F_{0}$ and $G_{0}$; as $F, G$ represent a linear series of dimension 1 , we may further assume that they were chosen so that $F_{0}, G_{0}$ defines a non-constant function. Then let $H_{1}$ and $H_{2}$ be inseparable polynomials of degree strictly less than $\bar{F}_{0}$ and $\bar{G}_{0}$, respectively, such that $\bar{F}_{0} H_{2}-\bar{G}_{0} H_{1}=1$ (this is possible by dividing the exponents $\bar{F}_{0}$ and $\bar{G}_{0}$ by $p$, applying Euclid's algorithm in $k[x]$, and multiplying all exponents by $p$ ). We now construct a new family $\tilde{F} / \tilde{G}$ over Spec $A$ as follows: if we denote by $\nu$ the map from $A[x]$ to itself which simply factors out common powers of $t$, then $\tilde{F}:=\nu\left(F \bar{G}_{0}-G \bar{F}_{0}\right)$, and $\tilde{G}:=F H_{2}-G H_{1}$. It is easy to check that applying an inseparable fractional linear transformation to $F / G$ will change $(d F) G-F(d G)$ by the 


\section{B. OSSERMAN}

determinant of the transformation, so it follows that $(d \tilde{F}) \tilde{G}-\tilde{F}(d \tilde{G})$ is the same as $(d F) G-F(d G)$, but with a positive power of $t$ factored out.

At $t=0$, we note that as we had $\bar{F}_{0} H_{2}-\bar{G}_{0} H_{1}=1, \tilde{G}$ is made up precisely of the common factors of $F_{0}$ and $G_{0}$, of which there can be at most $d-\operatorname{deg} f_{0} \leqslant d-p$. As we removed a positive power of $t$ from $(d F) G-F(d G)$, if we still have an inseparable limit, we can repeat the process as many times as necessary to remove all the powers of $t$ and obtain a separable limit. Each time we do, the degree of the denominator at $t=0$ clearly remains at most $d-p$. We thus end up with a family $\tilde{F} / \tilde{G}$ which over the generic fiber has the same different as $F / G$ away from infinity. If we let $K$ be the fraction field of $A$, we also note that we must have that the ideal generated by $\tilde{F}, \tilde{G}$ in $K[x]$ is the same as that generated by $F, G$. As $F, G$ had no common factors over $K$, it follows that $\tilde{F}, \tilde{G}$ have no common factors either. Now, as we have no common factors, we find by considering differents that away from $t=0$ (that is, at the generic fiber), $\tilde{F} / \tilde{G}$ has the same ramification as $F / G$ except possibly at infinity, as all the $e_{i}$ were specified to be less than $p$.

Denote by $\tilde{F}_{0}, \tilde{G}_{0}$ the polynomials obtained from $\tilde{F}, \tilde{G}$ at $t=0$. We claim that the different of $\left(\tilde{F}_{0}, \tilde{G}_{0}\right)$ away from infinity is the limit of the different of $f_{t}$, and in particular has degree $2 d-2$. Indeed, this follows from our hypothesis that the $P_{i}$ stay away from infinity, because when the limit is separable, the limit of the different is the different of the limit, with orders adding when points come together. Next, from our hypotheses, the different in the limit has order less than $p$ except at infinity, and we need not worry about wild ramification. Denote by $e_{\infty}$ the ramification index of $\tilde{F}_{0} / \tilde{G}_{0}$ at infinity, and suppose that $p \mid e_{\infty}$. In this situation, we can replace $\tilde{F}_{0}$ by subtracting off an appropriate multiple of $x^{e_{\infty}} \tilde{G}_{0}$, which will decrease $e_{\infty}$ without affecting the ramification away from infinity. Repeating as necessary, we can require that $\tilde{F}_{0} / \tilde{G}_{0}$ be tamely ramified at infinity (hence everywhere), without changing its behavior away from infinity, or the degree of $\tilde{G}_{0}$.

If we denote the greater of the degrees of $\tilde{F}_{0}, \tilde{G}_{0}$ by $\tilde{d}$, we then have that

$$
2 \tilde{d}-2=2 d-2+e_{\infty}-1 .
$$

We find in particular that $\tilde{d} \geqslant d$, so as the degree of $\tilde{G}_{0}$ was strictly less than $d$, it follows that $\tilde{d}$ is simply the degree of $\tilde{F}_{0}$, and $e_{\infty}=\tilde{d}-d_{0}$, where $d_{0}$ is the degree of $\tilde{G}_{0}$. Write $d_{0}=d-m$ for some $m$, where from our earlier bound on the degree of our denominators, we know that $m \geqslant p$. We then find that $\tilde{d}=d+m-1$, and $e_{\infty}=2 m-1>p$.

Finally, we translate back into the language of maps, by removing common factors. As vanishing indices cannot drop under specialization, and the different of the limit is the limit of the different, it then follows that at any point $P$, we can acquire base points only if more than one ramification section converges to $P$, which is to say only if $P=\bar{P}_{j}=\bar{P}_{j^{\prime}}$. In this situation, we let $b$ be the number of common factors of $\tilde{F}_{0}, \tilde{G}_{0}$ at $\bar{P}_{j}=\bar{P}_{j^{\prime}}$. It now follows immediately that removing these common factors, we have constructed a function as claimed in the statement of the theorem.

Putting the theorem together with Proposition 5.4, and noting that there are only finitely many possibilities for $m$ and $b$, we conclude as follows.

COROLlary 6.2. In the situation of the preceding theorem, if the $\bar{P}_{i}$ are general, there cannot be any $f_{t}$ as described, having an inseparable limit.

\section{The degeneration argument}

We complete the proof of Theorem 1.4 in this section via a degeneration argument. The basic situation we will consider is the family arising as follows.

Inside of $\mathbb{P}^{1} \times \mathbb{P}^{1}$, take the family of hyperbolas given in affine coordinates by $x y=t$, degenerating at $t=0$ to the union of the $x$-axis and $y$-axis. For each $t \neq 0$, we get a smooth $\mathbb{P}^{1}$, and 


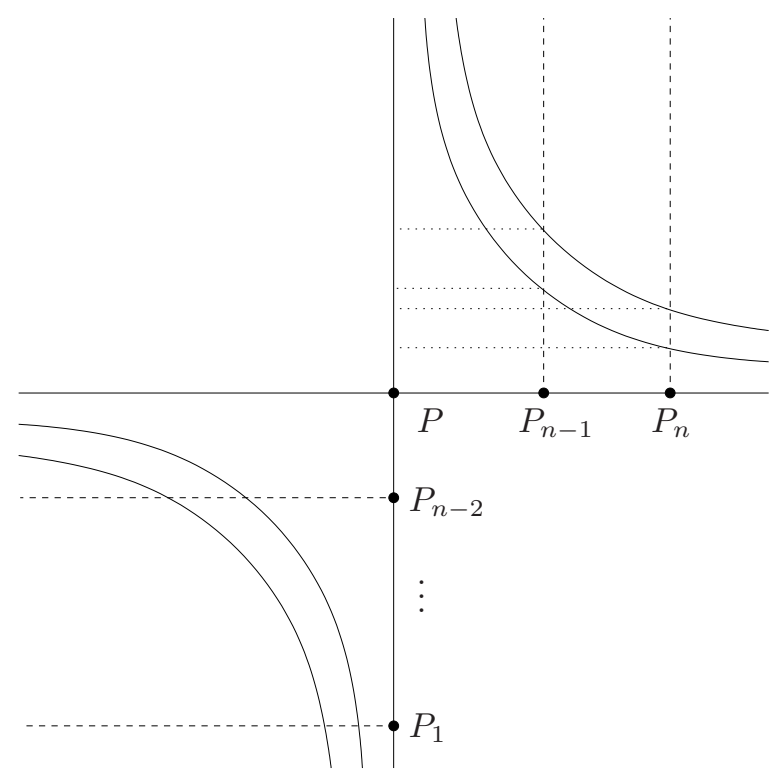

FiguRE 1. An explicit family of smooth rational curves degenerating to a node, with $n$ sections.

fix isomorphisms between them by projecting to the $y$-axis. Choose an isomorphism between our abstract $\mathbb{P}^{1}$ and the $y$-axis sending $P$ to the node; we can now speak of $P_{1}, \ldots, P_{n-2}$ as well as $P$ as fixed points on the $y$-axis and simultaneously on all the smooth fibers of our family; they are (constant) sections of our family. Now, choose any two points $P_{n-1}^{0}$ and $P_{n}^{0}$ on the $x$-axis away from 0 , and define sections $P_{n-1}^{t}$ and $P_{n}^{t}$ similarly via projection from our family to the $x$-axis rather than the $y$-axis. Under our fixed trivialization of the smooth fibers of the family, these sections both tend towards the section defined by $P$ (see Figure 1). We will consider this as a family $X$ over Spec $k[t]$, and write $X_{t}$ for the associated local family over Spec $k[t]_{(t)}$.

We briefly review the main concepts of the theory of limit linear series as it relates to our situation. See [Oss04c] for general definitions and, where applicable, proofs. On any non-singular fiber of our family, we know that a map to $\mathbb{P}^{1}$ (modulo automorphism of the image) corresponds to a $g_{d}^{1}$ on that fiber; we see that given a $g_{d}^{1}$ on the family away from the special fiber, we can obtain a $g_{d}^{1}$ on either the $x$ - or $y$-axis simply by projecting all fibers to the appropriate choice of axis. This pair gives the associated Eisenbud-Harris limit series on the nodal fiber; we have vanishing sequences $a_{i}^{x}$ and $a_{i}^{y}$ for $i=0,1$ at the node, and the degree of the induced map on the $x$-axis (respectively, $y$-axis) is at most $d-a_{0}^{x}$ (respectively, $d-a_{0}^{y}$ ), with the ramification index of the map at the node given by $a_{1}^{x}-a_{0}^{x}$ (respectively, $a_{1}^{y}-a_{0}^{y}$ ). We have the inequalities $a_{0}^{x}+a_{1}^{y} \geqslant d, a_{1}^{x}+a_{0}^{y} \geqslant d$; the data of a pair of $g_{d}^{1}$ 's on the components with vanishing sequences satisfying these inequalities is, in fact, the definition of an Eisenbud-Harris limit series, and we say that a given limit series is refined if these are both equalities.

Considering the ramification imposed on the induced maps on the $x$-axis and $y$-axis together, we find that we have

$\sum_{i}\left(e_{i}-1\right)+\left(a_{1}^{x}-a_{0}^{x}-1\right)+\left(a_{1}^{y}-a_{0}^{y}-1\right) \geqslant(2 d-2)+\left(2 d-2 a_{0}^{x}-2 a_{0}^{y}-2\right)=\left(2\left(d-a_{0}^{x}\right)-2\right)+\left(2\left(d-a_{0}^{y}\right)-2\right)$.

Hence, by Riemann-Hurwitz, if the limits are separable, we immediately conclude that we must have equality, so the limit forms a refined limit series. Furthermore, the maps cannot have any additional base points or ramification, so the corresponding maps must have degrees precisely $d-a_{0}^{x}$ and $d-a_{0}^{y}$, with ramification index $a_{1}^{x}-a_{0}^{x}=a_{1}^{y}-a_{0}^{y}$ at the node. 


\section{B. OSSERMAN}

Given these observations, our general theory, and specifically [Oss04c, Theorem 5.3], gives us the following.

Theorem 7.1. Associated to our families $X$ and $X_{t}$, and any choice of ramification indices $e_{i}$ such that $\sum_{i}\left(e_{i}-1\right)=2 d-2$, are schemes $G_{d}^{1}:=G_{d}^{1}\left(X,\left\{\left(P_{i}, e_{i}\right)\right\}_{i}\right)$ and $\tilde{G}_{d}^{1}:=G_{d}^{1}\left(X_{t},\left\{\left(P_{i}, e_{i}\right)\right\}_{i}\right)$, with the latter obtained from the former by base change, and the fibers parametrizing (limit) linear series with the required ramification on the fibers of $X$ and $X_{t}$. We also have open subschemes $G_{d}^{1 \text {,sep }}$ and $\tilde{G}_{d}^{1, \text { sep }}$ parametrizing limit series which are separable when restricted to every component of every fiber. Over $t=0, G_{d}^{1, \text { sep }}$ (equivalently, $\tilde{G}_{d}^{1, \mathrm{sep}}$ ) parametrizes simply Eisenbud-Harris limit series, and contains only refined limit series.

Proof. Most of this is immediate from [Oss04c, Theorem 5.3]. The fact that $G_{d}^{1, \text { sep }}$ parametrizes Eisenbud-Harris series on the special fiber follows from [Oss04c, Corollary 6.8] together with the assertion that the only separable Eisenbud-Harris limit series are refined, which we observed above.

Given this language, we can readily apply [Oss04c, Corollary 6.12] to obtain the following.

Corollary 7.2. With the notation of the above theorem, if $P_{1}, \ldots, P_{n-2}$ and $P$ are chosen generally, and $e_{n-1}+e_{n}<p$, then $\tilde{G}_{d}^{1, \text { sep }}$ is finite etale over Spec $k[t]_{(t)}$. In particular, it has the same number of points, all reduced, in the geometric generic and special fibers, and the fibers of $G_{d}^{1, \text { sep }}$ have the same number of points at general $t$ as at $t=0$.

Proof. First, the assertion on the fibers of $G_{d}^{1, \text { sep }}$ for general $t$ follows immediately from the statement on $\tilde{G}_{d}^{1, \text { sep }}$, together with the fact that $\tilde{G}_{d}^{1, \text { sep }}$ is obtained from $G_{d}^{1, \text { sep }}$ simply by localization of the base around $t=0$.

Next, to obtain the desired statement on $\tilde{G}_{d}^{1, \text { sep }}$, we need only verify that the conditions (I)-(III) of [Oss04c, Corollary 6.12] are satisfied: first, that every separable Eisenbud-Harris limit series on the special fiber is refined; second, that the scheme of separable Eisenbud-Harris limit series on the special fiber consists of a finite number of reduced points; and third, that if $A$ is a DVR, any $A$-valued point of $\tilde{G}_{d}^{1}$ mapping flatly to Spec $k[t]_{(t)}$ and being separable at the generic point is also separable on the closed point. Condition (I) is satisfied even without the generality hypothesis, as stated in the above theorem.

Condition (III) is for the most part simply an application of Corollary 6.2; indeed, given an $A$-valued point of $\tilde{G}_{d}^{1}$ flat over Spec $k[t]_{(t)}$, projection to the $y$-axis would give a family of $g_{d}^{1}$ 's on $\mathbb{P}^{1}$ with ramification sections specializing to the $P_{1}, \ldots, P_{n-2}, P$, which are general by hypothesis. Then Corollary 6.2 says that if the family is generically separable, it must remain separable on the special fiber. It remains to see that the same holds if we project to the $x$-axis. For this, considering the different we note that the vanishing sequence on the $y$-axis at the node will satisfy $a_{0}^{y}+a_{1}^{y}-1=$ $e_{n-1}+e_{n}-2$, and in particular $a_{1}^{y}<p$. On the other hand, $a_{0}^{x}+a_{1}^{y} \geqslant d$, so as $a_{0}^{x}$ is the number of base points acquired on the $x$-axis, the degree on the $x$-axis is less than or equal to $d-a_{0}^{x} \leqslant a_{1}^{y}<p$, and we also cannot have an inseparable limit along the $x$-axis, giving condition (III).

Lastly, we prove the validity of condition (II) by induction on $n$. The basic observation is because the space of refined Eisenbud-Harris limit series may be viewed simply as a disjoint union over all vanishing sequences satisfying $a_{i}^{x}+a_{1-i}^{y}=d$ of the products of the schemes parametrizing $g_{d}^{1}$ 's with appropriate ramification on each component, it suffices to see that these latter are made up of reduced points. It is easy to see that as the vanishing sequences vary, if we simply remove the base points $a_{0}^{x}$ and $a_{0}^{y}$, we will have the same ramification index $e$ at the node on each component, the degrees on each component will be such that the expected dimension (taking $e$ into account as well as the $e_{i}$ ) will be zero, and $e$ will vary arbitrarily given this constraint, together with the constraint that 


\section{RATIONAL FUnCTIONS WITH GIVEN RAMIFICATION IN CHARACTERISTIC $p$}

the degrees on each component be at most $d$. In particular, it suffices to see that for points chosen generally, the scheme of separable $g_{d}^{1}$ 's in the $(n-1)$-point and three-point cases always consist of a finite number of reduced points, and by induction on the statement of our corollary, it is enough to see this in the three-point case, which we have conveniently already handled in Theorem 4.2.

We are now ready for the proof of Theorem 1.4.

Proof of Theorem 1.4. First, the second form of the $n>3$ case is easily checked by induction to be equivalent to the recursive formula. Also, we already have the $n=3$ formula from Theorem 4.2.

For the recursive formula, we may assume that $e_{n-1}+e_{n}<p$, thanks to Corollary 5.3. By Corollary 7.2, for all our points chosen generally, and a general choice $t, G_{d}^{1, \text { sep }}$ has the same number of points over that particular $t$ as it does over $t=0$. This sets up a simple recursion formula to calculate $N_{\text {gen }}\left(\left\{e_{i}\right\}_{i}\right)$ : the number will be given by the number over the special fiber, which is the sum over all choices $e$ of ramification index at the node of $N_{\text {gen }}\left(e, e_{n-1}, e_{n}\right) N_{\text {gen }}\left(\left\{e_{i}\right\}_{i<n-1}, e\right)$.

We recall that the formula we wanted to prove for Theorem 1.4 was

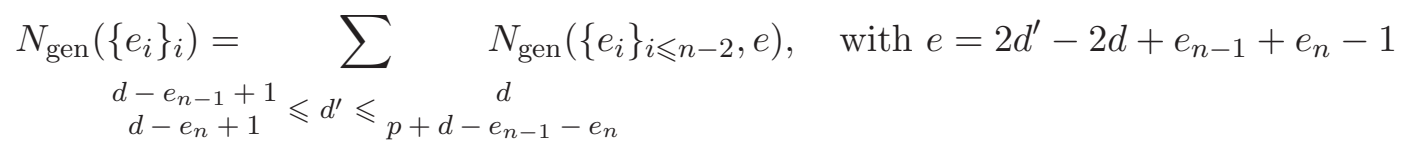

and that in the proof of Corollary 5.3 we saw that the above inequalities for $d^{\prime}$ were equivalent to the following inequalities on $e$ :

$$
\begin{aligned}
& e_{n}-e_{n-1}+1 \\
& e_{n-1}-e_{n}+1
\end{aligned} \leqslant e \leqslant \begin{gathered}
e_{n}+e_{n-1}-1 \\
2 p-1-e_{n-1}-e_{n}
\end{gathered} .
$$

We first show that the above inequalities for $e$ are precisely the range for which $N_{\text {gen }}\left(e, e_{n-1}, e_{n}\right)$ $=1$. However, with Theorem 4.2 at our disposal, this is a trivial observation, as $e_{n}-e_{n-1}+1 \leqslant e$, $e_{n-1}-e_{n}+1 \leqslant e$, and $e \leqslant e_{n}+e_{n-1}-1$ are precisely the inequalities insuring that the ramification indices are less than the degree of the map, and $e \leqslant 2 p-1-e_{n-1}-e_{n}$ insures that the degree is less than $p$. Finally, we need to know that the degree on the three-point component will be less than $d$. This degree will be given by $\left(e+e_{n-1}+e_{n}-1\right) / 2$, so we find that a priori, we need $e \leqslant 2 d-e_{n-1}-e_{n}+1$. However, we note that the right-hand side is actually $2 d^{\prime}-e$, so this inequality is equivalent to $e \leqslant d^{\prime}$, and we need not include it with the conditions, as if it is violated we will have $N_{\text {gen }}\left(\left\{e_{i}\right\}_{i \leqslant n-2}, e\right)=0$, and there will be no contribution to the sum. This completes the proof of our main theorem.

\section{Examples and further questions}

We first apply our main theorem in the case of four points. For a given $d^{\prime}$ as in Theorem 1.4, $N_{\text {gen }}\left(e_{1}, e_{2}, e\right)=1$ if $e_{1}, e_{2}, e \leqslant d^{\prime}$ and $p>d^{\prime}$, and $N_{\text {gen }}\left(e_{1}, e_{2}, e\right)=0$ otherwise. Rewriting this condition in terms of $d^{\prime}$, we get the bounds $e_{1} \leqslant d^{\prime}, e_{2} \leqslant d^{\prime}, d^{\prime} \leqslant 2 d-e_{3}-e_{4}+1, d^{\prime} \leqslant p-1$, and including these bounds for $d^{\prime}$ along with those of Theorem 1.4, simply by substracting the various bounds for possible values of $d^{\prime}$ we obtain the following.

Corollary 8.1. The number $N_{\text {gen }}\left(\left\{e_{i}\right\}_{i}\right)$ of self-maps of $\mathbb{P}^{1}$ of degree $d$ in characteristic $p$, ramified to orders $e_{1}, \ldots, e_{4}$ at four general points, with each $e_{i}<p$ and $2 d-2=\sum_{i}\left(e_{i}-1\right)$, and counted modulo automorphism of the image, is given by the formula

$$
N_{\text {gen }}\left(\left\{e_{i}\right\}_{i}\right)=\min \left\{\left\{e_{i}\right\}_{i},\left\{d+1-e_{i}\right\}_{i},\left\{p-e_{i}\right\}_{i},\left\{p-d-1+e_{i}\right\}_{i}\right\},
$$

or equivalently,

$$
N_{\text {gen }}\left(\left\{e_{i}\right\}_{i}\right)=\min \left\{\left\{e_{i}\right\}_{i},\left\{d+1-e_{i}\right\}_{i}\right\}-\max \{0, d+1-p\} .
$$

Further, all of these maps are without any non-trivial first-order deformations. 


\section{B. OSSERMAN}

Example 8.2. We explore an example which demonstrates all the basic behaviors we have described so far, and may be solved explicitly: maps of degree 3 , with four simple ramification points. We may assume without loss of generality that $P_{1}=0, P_{2}=\infty, P_{3}=1$, and we let $P_{4}$ be a general parameter $\lambda$. We see immediately that our four ramification points must have distinct images, so we may further specify that our maps fix $P_{1}$ and $P_{2}$, from which we deduce that they are of the form $f=x^{2}(a x+b) /(x+c)$, with $a, b, c$ all non-zero. As we did not specify that $P_{3}$ be fixed, we have one remaining degree of freedom, and may set $b=1$. Now, if we consider the zeroes and poles of $d f$, we can calculate directly that our possible maps satisfy $2 c=2 a \lambda$ and $1+3 a c=-(1+\lambda) 2 a$, which in characteristic other than 2 means $c$ is determined by $a$ and $\lambda$, and $a$ satisfies $3 \lambda a^{2}+2(1+\lambda) a+1=0$. In characteristic 3 , we get a unique (separable) solution, while in characteristics 0 or $p>3$, we get two solutions for general $\lambda$. We find that these solutions come together when $1-\lambda+\lambda^{2}=0$. Finally, in characteristic 3 , we also see that the unique solution $f=\left(x^{3}+(1+\lambda) x^{2}\right) /((1+\lambda) x+\lambda)$ specializes to an inseparable solution when $\lambda$ goes to -1 .

Remark 8.3. In the context of covers with prescribed branching, there is a very general result on lifting to characteristic 0; see [BLR98, Ch. 11, Proof of Proposition 5.1]. One can use this and the three-transitivity of Aut $\left(\mathbb{P}^{1}\right)$ to prove a similar result for lifting $g_{d}^{1}$ 's on $\mathbb{P}^{1}$ with prescribed ramification at three points, and one might be tempted to conjecture that one can generalize to arbitrary numbers of points and tame ramification on $\mathbb{P}^{1}$. However, Proposition 5.4 shows that such a statement cannot hold even in this case, as by virtue of Corollary 3.2 only finitely many of the infinitely many constructed maps would be able to lift to characteristic 0 . However, it may still be true that one can prove such a statement in the high and mid characteristic ranges. Indeed, we are able to prove this in more generality in [Oss04c, Corollary 5.5], subject to an expected-dimension hypothesis. In particular, in our case of self-maps of $\mathbb{P}^{1}$, we can conclude thanks to Theorem 3.3 that lifting to characteristic 0 is always possible for tame ramification indices and general ramification points, or in high characteristic and arbitrary ramification points, and we have reduced the midcharacteristic case down to the question of finiteness for arbitrary distinct ramification points.

We conclude with some further questions. We could reasonably start with remaining questions about the case of $\mathbb{P}^{1}$, including the following.

Question 8.4. Is it true that for a given $d$ and $e_{i}$, the number of maps is either always finite or always infinite as the $P_{i}$ are allowed to move? Can we prove that it is always finite in the mid-characteristic case?

Question 8.5. What happens in low characteristic when more than one ramification index is greater than $p$ ?

Question 8.6. What can we say about the dimension of spaces of wildly ramified maps? When do wildly ramified maps exist for general ramification points?

This first question is partially answered by applying results of Mochizuki in [Oss04e], and similar arguments may be expected to give a complete affirmative answer. The last question is explored further in [Oss05a]. We briefly examine some examples of the second question. The following lemma is trivial.

Lemma 8.7. Let $f, g$ be rational functions on a smooth curve $C$, and $P \in C$ a point where both $f$ and $g$ are defined. Then $f+g$ has ramification index at $P$ at least as large as $f$ if and only if the ramification index of $g$ at $P$ is at least as large as that of $f$.

In particular, in the case that $C=\mathbb{A}^{1}$ and $f$ is a tamely ramified polynomial of degree $d$, with $d>p$ and prime to $p$, we have that $f+g$ is another polynomial of degree $d$, with the same ramification as $f$, if and only if $g$ is an inseparable polynomial of degree less than $d$, and with ramification index greater than that of $f$ at every point of $\mathbb{A}^{1}$. 


\section{RATIONAL FUnCTIONS WITH GIVEN RAMIFICATION IN CHARACTERISTIC $p$}

Example 8.8. We conclude that if $e_{1}=d$ is strictly between $2 p$ and $3 p$, for some choices of $e_{i}$ we have a positive-dimensional family of maps with the given ramification, while for others we only have finitely many such maps. For instance, if we have $p<e_{2}<2 p$, but $e_{i}<p$ for all $i>2$, we obtain a unique inseparable polynomial (up to fractional linear transformation) of degree $2 p$ ramified to order $2 p$ at $P_{2}$, which gives us a one-dimensional family of maps with the desired ramification. However, if $e_{2}>2 p$, or if $e_{3}$ is also between $p$ and $2 p$, no inseparable polynomial of the specified form exists, so our separable map is necessarily unique. Furthermore, these examples are non-vacuous: examples of the first and second may be obtained simply by $x^{d}-x^{e_{2}}$, for $e_{1}-e_{2} \neq p$, while $2 x^{2 p+1}-x^{2 p}-2 x^{p+1}$ is an example of the third.

Lastly, one could ask the same questions about maps from higher-genus curves to $\mathbb{P}^{1}$. These have been answered in the case of characteristic 0 in [Oss03], and the argument there would also apply in characteristic $p$ given an appropriate generalization of Theorem 6.1 to control the possibility of separable maps specializing to inseparable maps. The case of higher-dimensional linear series is still open as well, even in characteristic 0 .

\section{Appendix. Moduli schemes of ramified maps}

The goal of this appendix is to construct moduli schemes of maps of curves required to have at least given ramification, but at unspecified points. Before we begin, we recall the well-known corollary of Grothendieck's work on the Hilbert scheme.

Theorem A.1. Given $X$ and $Y$ two smooth, projective, geometrically connected curves over a locally Noetherian scheme $S$ and a positive integer $d$, then the functor $\mathcal{M o r}_{S}^{d}(X, Y)$ parametrizing degree $d$ morphisms from $X$ to $Y$ over $S$ is representable by a quasi-projective scheme. In particular, $\mathcal{A} u t_{S}(X)=\operatorname{Mor}_{S}^{1}(X, X)$ is representable.

Proof. Without the degree hypothesis, the functor is constructed in [Gro61, pp. 221-220] (where it is called Hom) as an open subscheme of the Hilbert scheme via the graph associated to a morphism. Now, if $\mathcal{L}$ and $\mathcal{M}$ are ample line bundles on $X$ and $Y$, and $f$ a morphism of degree $d$, one checks that the Hilbert polynomial of the graph under the projective imbedding of $X{ }_{S} Y$ induced by $\pi_{1}^{*} \mathcal{L} \otimes \pi_{2}^{*} \mathcal{M}$ is uniquely determined by $d$, so the Mor scheme is naturally a disjoint union over all $d$ of schemes representing $\operatorname{Mor}^{d}$, each of which is quasi-projective.

In order to see that $\mathcal{A} u t_{S}(X)=\mathcal{M o r}_{S}^{1}(X, X)$, we first note that for any $d>0, \operatorname{Mor}_{S}^{d}(X, Y)$ consists entirely of scheme-theoretically surjective morphisms. Indeed, given $f \in \mathcal{M o r}^{d}$, one checks by the criterion on flatness and fibers (see [GD66, Theorem 11.3.10]) that $f$ is faithfully flat, which implies scheme-theoretic surjectivity. Now, to see that $\mathcal{A} u t_{S}(X)=\mathcal{M o r}_{S}^{1}(X, X)$, it suffices to note that in our situation, one can check whether $f$ is a closed immersion on each fiber $f_{s}$ (see [GD61, Proposition 4.6.7]), so the desired assertion follows from the well-known case of smooth curves over $S=\operatorname{Spec} k$ (see, for instance, [GD61, Corollary 4.4.9]).

We also have the following.

Proposition A.2. With the notation of the preceding theorem, there exists an open subscheme $\operatorname{Mor}_{S}^{d, \text { sep }}(X, Y)$ of $\operatorname{Mor}_{S}^{d}(X, Y)$ parametrizing morphisms which are separable on every fiber.

Proof. Let $M:=\operatorname{Mor}_{S}^{d}(X, Y), X_{M}$ and $Y_{M}$ be the pullbacks of $X$ and $Y$ to $M, \tilde{f}: X_{M} \rightarrow Y_{M}$ be the universal morphism of degree $d$, defined over $M$; we get an induced map $\tilde{f}^{*} \Omega_{Y_{M} / M}^{1} \rightarrow \Omega_{X_{M} / M}^{1}$ of line bundles on $X_{M}$, with the cokernel giving the locus on $X_{M}$ where $\tilde{f}$ is ramified. The complement is an open set, and its image in $M$ is clearly the locus of separable maps; as $X$ is flat and of finite type over $S, X_{M}$ is flat and of finite type over $M$, and in particular open, so we have constructed an open subscheme of $M$ corresponding to separable maps, as desired. 


\section{B. Osserman}

We also recall a standard construction involving the jet bundle, or bundle of principal parts $\mathcal{P}_{X / S}^{n}$, associated to an $S$-scheme $X$. The terminology and notation is not standard, however.

Definition A.3. We define the nth cotangent bundle $\Upsilon_{X / S}^{n}$ to be the kernel of the natural map $\mathcal{P}_{X / S}^{n} \rightarrow \mathcal{O}_{X}$; explicitly, consider $\mathcal{O}_{X} \otimes_{\mathcal{O}_{S}} \mathcal{O}_{X}$ as an $\mathcal{O}_{X}$-module via left multiplication, and consider the natural map to $\mathcal{O}_{X}$ sending $a \otimes b$ to $a b$. Then if we denote the kernel of this map by $\mathcal{I}_{X / S}$, with the induced $\mathcal{O}_{X}$-module structure, $\Upsilon_{X / S}^{n}:=\mathcal{I}_{X / S} / \mathcal{I}_{X / S}^{n+1}$.

We recall the following proposition.

Proposition A.4. With notation as in the preceding definition:

(i) $\Upsilon_{X / S}^{n}$ is compatible with base change;

(ii) on affine opens $U, \mathcal{I}_{X / S}$ is generated by elements of the form $a \otimes 1-1 \otimes a$, for $a \in \mathcal{O}_{X}(U)$;

(iii) if $X$ is smooth over $S, \Upsilon_{X / S}^{n}$ is locally free.

Proof. Compatibility with base change for $\mathcal{P}_{X / S}^{n}$ is [GD67, Proposition 16.4.5]; because $\Upsilon_{X / S}^{n}$ is the kernel of a map (clearly compatible with base change) to $\mathcal{O}_{X}$, and $\mathcal{O}_{X}$ is free, it follows that $\Upsilon_{X / S}^{n}$ is compatible with base change. Part (ii) is [GD64, Lemma 0.20.4.4]. Finally, part (iii) follows from the same statement for $\mathcal{P}_{X / S}^{n}$, which is [GD67, Proposition 17.12.4], as $\Upsilon_{X / S}^{n}$ is the kernel of a surjective map from $\mathcal{P}_{X / S}^{n}$ to $\mathcal{O}_{X}$ (in fact, this is somewhat gratuitous, as the argument for $\mathcal{P}_{X / S}^{n}$ works without modification for $\left.\Upsilon_{X / S}^{n}\right)$.

We now specify in full detail the functor we wish to represent.

Definition A.5. Suppose that we are given a pair of smooth, projective, geometrically connected curves $X, Y$ over a locally Noetherian base $S$, as well as $n$ integers $e_{i}$, and $d \geqslant 1$. Then the functor $\mathcal{M R}_{S}^{d}\left(X, Y,\left\{e_{i}\right\}_{i}\right)$ associates to any scheme $T$ over $S$ the set of separable morphisms $f$ from $X_{T}$ to $Y_{T}$ over $T$ of degree $d$, together with a choice of $n$ disjoint $T$-valued points $P_{i}$ of $X_{T}$, such that the fiber of $f\left(P_{i}\right)$ contains an $e_{i}$ th-order thickening of $P_{i}$ inside of $X_{T}$ for each $i$.

Conceptually, this functor is the functor of maps $f$ of degree $d$ between $X$ and $Y$, together with points $P_{i}$ on $X$ which are (at least) $e_{i}$ th-order ramification points of $f$.

Our main result is the following.

Theorem A.6. The functor $\mathcal{M R}=\mathcal{M R}_{S}^{d}\left(X, Y,\left\{e_{i}\right\}_{i}\right)$ is representable by a scheme $M R$. We also have the natural data of morphisms ram $: M R \rightarrow X^{n}$ and branch $: M R \rightarrow Y^{n}$ and actions of the group schemes $\operatorname{Aut}(X)$ and $\operatorname{Aut}(Y)$ on $M R$ over $Y^{n}$ and $X^{n}$ respectively. Furthermore, Aut $(Y)$ acts freely on $M R$.

Proof. First we note that all of the assertions other than representability can be verified simply on the functor level: the morphism ram is the forgetful transformation which takes a point of $M R$ and remembers only the $P_{i}$; similarly, the morphism branch remembers the $f\left(P_{i}\right)$, which are sections of $Y_{T}$. A point $g$ of $\operatorname{Aut}(Y)$ act on points of $M R$ by sending $f$ to $g \circ f$ and leaving the $P_{i}$ fixed, and similarly $g \in \operatorname{Aut}(X)$ acts on $M R$ by sending $f$ to $f \circ g$ and the $P_{i}$ to $g^{-1} P_{i}$, which fixes $f\left(P_{i}\right)$. The freeness of the $\operatorname{Aut}(Y)$ action follows easily from the statement that any point of $M R$ corresponds to a scheme-theoretically surjective map, noted in the proof of Theorem A.6.

Clearly, we have a forgetful map from $M R$ to $M=\operatorname{Mor}^{d, \operatorname{sep}}(X, Y)$; as the latter is representable, it will be enough to show that the map of functors is also representable. In fact, if we use the convention that $X_{M}^{n}$ denotes the product of $n$ copies of $X_{M}$ over $M$, the sections in the definition of our functor will allow us to describe $M R$ as a closed subscheme of $X_{M}^{n}$ with the pairwise diagonals removed. We claim that it is enough to handle the case $n=1$ : suppose that we have done this case, 


\section{RATIONAL FUnCTIONS WITH GIVEN RAMIFICATION IN CHARACTERISTIC $p$}

and for each $i$ let $M R_{i}$ be the resulting scheme; then if we imbed the product of the $M R_{i}$ as a closed subscheme of $X_{M}^{n}$, and remove pairwise diagonals, we get the desired scheme.

As $X$ and $Y$ are smooth over $S$ by hypothesis, $\Upsilon_{X / S}^{e-1}$ and $\Upsilon_{Y / S}^{e-1}$ are locally free, so the kernel of any morphism $f^{*} \Upsilon_{Y / S}^{e-1} \rightarrow \Upsilon_{X / S}^{e-1}$ is representable by a closed subscheme of $X$ over $S$, and the following lemma completes the proof of our theorem.

Lemma A.7. Let $f: X \rightarrow Y$ be a morphism of separated $S$-schemes. Then there is a natural map $f^{*} \Upsilon_{Y / S}^{e-1} \rightarrow \Upsilon_{X / S}^{e-1}$ such that for any $T$ over $S$, and any section $\sigma: T \rightarrow X_{T}$ we have $\left(f^{*} \Upsilon_{Y / S}^{e-1} \rightarrow\right.$ $\left.\Upsilon_{X / S}^{e-1}\right)_{\sigma(T)}=0$ if and only if the fiber of $f_{T}$ over $f_{T}(\sigma(T))$ contains an eth order thickening of $\sigma(T)$ inside $X_{T}$.

Proof. The map from $f^{*} \Upsilon_{Y / S}^{e-1} \rightarrow \Upsilon_{X / S}^{e-1}$ is simply that induced by $f^{*} \otimes f^{*}: f^{-1} \mathcal{O}_{Y} \otimes_{\mathcal{O}_{S}} f^{-1} \mathcal{O}_{Y} \rightarrow$ $\mathcal{O}_{X} \otimes_{\mathcal{O}_{S}} \mathcal{O}_{X}$. Our assertion is local, so we immediately reduce to affines, and consider the situation that $X_{T}=\operatorname{Spec} A, Y_{T}=\operatorname{Spec} B$, and $T=\operatorname{Spec} R$. As $X$ and $Y$ are separated over $S$, a section is a closed immersion, so we also denote by $I_{\sigma}$ the ideal corresponding to $\sigma(T)$ in $X_{T}$, and set $I_{\sigma}^{\prime}:=I_{\sigma} \otimes_{R} A \subset A \otimes_{R} A$. Now, the fiber of $f(\sigma(T))$ is given by $\operatorname{Spec}\left(A / I_{\sigma} \otimes_{B} A\right)$, cut out in $X_{T}$ by the ideal of $A$ generated by $\left(f_{T}^{*} B\right) \cap I_{\sigma}$. The fiber contains an eth order thickening of $\sigma_{T}$ if and only if this ideal is contained in $I_{\sigma}^{e}$, which is to say, if and only if $\left(f_{T}^{*} B\right) \cap I_{\sigma} \subset I_{\sigma}^{e}$. We claim that this is equivalent to $1 \otimes\left(\left(f_{T}^{*} B\right) \cap I_{\sigma}\right) \subset\left(I_{\sigma}^{\prime}, A \otimes I_{\sigma}^{e}\right)$ : indeed, this follows immediately from the fact that $\left(A \otimes_{R} A\right) /\left(I_{\sigma}^{\prime}, A \otimes I_{\sigma}^{e}\right) \cong A / I_{\sigma} \otimes R A / I_{\sigma}^{e} \cong A / I_{\sigma}^{e}$.

On the other hand, $\left(f^{*} \Upsilon_{Y / S}^{e-1} \rightarrow \Upsilon_{X / S}^{e-1}\right)_{\sigma(T)}=0$ if and only if $\left(f_{T}^{*} \Upsilon_{Y_{T} / T}^{e-1} \rightarrow \Upsilon_{X_{T} / T}^{e-1}\right)_{\sigma(T)}=0$, by Proposition A.4(i), and this is equivalent to the assertion that $f_{T}^{*} \mathcal{I}_{Y_{T} / T}$ is contained in the ideal generated by $I_{\sigma}^{\prime}$ and $\mathcal{I}_{X_{T} / T}^{e}$. Thus, the proof of the lemma is reduced to the following two assertions: first, that modulo $I_{\sigma}^{\prime}$, we have $f_{T}^{*} \mathcal{I}_{Y_{T} / T}=1 \otimes\left(\left(f_{T}^{*} B\right) \cap I_{\sigma}\right)$; and second, that $\left(I_{\sigma}^{\prime}, \mathcal{I}_{X_{T} / T}^{e}\right)=$ $\left(I_{\sigma}^{\prime}, A \otimes_{R} I_{\sigma}^{e}\right)$. By Proposition A.4(ii), $\mathcal{I}_{X_{T} / T}$ and $\mathcal{I}_{Y_{T} / T}$ are generated by elements of the form $a \otimes 1-1 \otimes a$ and $b \otimes 1-1 \otimes b$, respectively. The main observation is that because $I_{\sigma}$ is the ideal of a section, to generate these ideals it suffices to restrict to $a$ with $a \in I_{\sigma}$, and to $b$ with $f_{T}^{*} b \in I_{\sigma}$. The latter immediately gives the first assertion. The second assertion also follows easily, as we then have that $\mathcal{I}_{X_{T} / T}^{e}$ is generated by products $\prod_{j \leqslant e}\left(i_{j} \otimes 1-1 \otimes i_{j}\right)$, which are equivalent modulo $I_{\sigma}^{\prime}$ to elements of $1 \otimes I_{\sigma}^{e}$, as desired.

Remark A.8. The $\operatorname{Aut}(X)$ action is not free in general, often having a non-trivial finite sub-group scheme stabilizing any given morphism. However, it is easy enough to see that the stabilizer of any $k$-valued point $f \in M R$ is in fact a finite group scheme. Indeed, in this case, we may as well set $S=\operatorname{Spec}(k)$. As $\operatorname{Aut}(X)$ is a finite-type group scheme, the stabilizer will likewise be a finite-type group scheme over $k$, and it thus suffices to show that it consists of only finitely many $\bar{k}$-valued points. Now, an automorphism of $X_{\bar{k}}$ is determined on the generic point, and will have to fix $K\left(Y_{\bar{k}}\right)$ inside $K\left(X_{\bar{k}}\right)$ in order to fix $f$; as $K\left(Y_{\bar{k}}\right)$ is a finite subfield of $K\left(X_{\bar{k}}\right)$, the relevant automorphism group is finite, and we conclude the desired assertion.

\section{ACKnowledgements}

I would like to thank Johan de Jong for his tireless and invaluable guidance. I would also like to thank Joe Harris, Dan Laksov, Astrid Giugni, and Roya Beheshti for their helpful discussions.

\section{REFERENCES}

Bel06 P. Belkale, Geometric proofs of Horn and saturation conjectures, J. Algebraic Geom. 15 (2006), 133-173. 


\section{RATIONAL FUnCTIONS WITH GIVEN RAMIFICATION IN CHARACTERISTIC $p$}

BLR98 J.-B. Bost, F. Loeser and M. Raynaud (eds), Courbes semi-stables et groupe fondamental en géométrie algébrique (Birkhäuser, Basel, 1998).

EH83 D. Eisenbud and J. Harris, Divisors on general curves and cuspidal rational curves, Invent. Math. 74 (1983), 371-418.

EH86 D. Eisenbud and J. Harris, Limit linear series: Basic theory, Invent. Math. 85 (1986), 337-371.

Ful98 W. Fulton, Intersection theory, second edition (Springer, Berlin, 1998).

Gol91 L. R. Goldberg, Catalan numbers and branched coverings by the Riemann sphere, Adv. Math. 85 (1991), 129-144.

Gro61 A. Grothendieck, Techniques de construction et théorèmes d'existence en géométrie algébrique, IV: Les schémas de Hilbert, Semin. Bourbaki vol. 6, exp. 221 (Soc. Math. France, Paris, 1961), 249-276.

GD61 A. Grothendieck and J. Dieudonné, Éléments de géométrie algébrique: III. Étude cohomologique des faisceaux cohérents, I, Publ. Math. Inst. Hautes Études Sci. 11 (1961).

GD64 A. Grothendieck and J. Dieudonné, Éléments de géométrie algébrique: IV. Étude locale des schémas et des morphismes de schémas, I, Publ. Math. Inst. Hautes Études Sci. 20 (1964).

GD66 A. Grothendieck and J. Dieudonné, Éléments de géométrie algébrique: IV. Étude locale des schémas et des morphismes de schémas, III, Publ. Math. Inst. Hautes Études Sci. 28 (1966).

GD67 A. Grothendieck and J. Dieudonné, Éléments de géométrie algébrique: IV. Étude locale des schémas et des morphismes de schémas, IV, Publ. Math. Inst. Hautes Études Sci. 32 (1967).

Log03 A. Logan, The Kodaira dimension of moduli spaces of curves with marked points, Amer. J. Math. 125 (2003), 105-138.

MV02 E. Mukhin and A. Varchenko, Solutions to the XXX type Bethe ansatz equations and flag varieties, Cent. Eur. J. Math. 1 (2003), 238-271.

Oss03 B. Osserman, The number of linear series on curves with given ramification, Int. Math. Res. Not. 47 (2003), 2513-2527.

Oss04a B. Osserman, Frobenius-unstable bundles and p-curvature, Trans. Amer. Math. Soc., to appear. arXiv:math.AG/0409266.

Oss04b B. Osserman, The generalized Verschiebung map for curves of genus 2, Preprint (2004), arXiv:math.AG/0410613.

Oss04c B. Osserman, A limit linear series moduli scheme, Ann. Inst. Fourier (Grenoble), to appear. arXiv:math.AG/0407496.

Oss04d B. Osserman, Logarithmic connections with vanishing p-curvature, Preprint (2004), arXiv:math.AG/0409145.

Oss04e B. Osserman, Mochizuki's crys-stable bundles: A lexicon and applications, Preprint (2004), arXiv:math.AG/0410323.

Oss05a B. Osserman, Deformations of covers, Brill-Noether theory, and wild ramification, Math. Res. Lett. 12 (2005), 483-491.

Oss05b B. Osserman, Linear series and existence of branched covers, Preprint (2005), arXiv:math.AG/0507096.

Sch02 I. Scherbak, Rational functions with prescribed critical points, Geom. Funct. Anal. 12 (2002), $1365-1380$.

Vak03 R. Vakil, Schubert induction, Ann. of Math. (2), to appear. arXiv:math.AG/0302296.

Brian Osserman osserman@math.berkeley.edu

Department of Mathematics, University of California, Berkeley, CA 94720-3840, USA 